\title{
Agrimonitor, herramienta del banco internacional de desarrollo para políticas agropecuarias en el Ecuador.
}

DOI: https://doi.org/10.33262/ap.v3i3.2.94

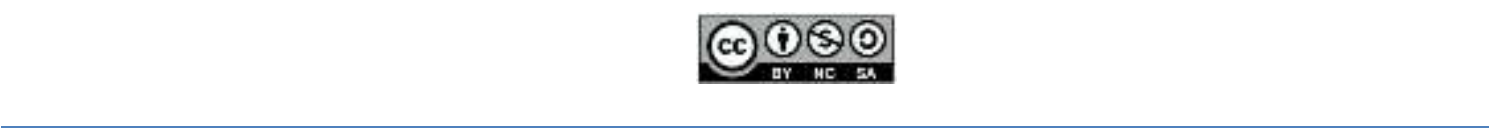

Agrimonitor, tool of the international development bank for agricultural policies in ecuador.

Alberto Esteban Mogrovejo Lazo. ${ }^{1}$, Román Florencio Carabajo Alvear. ${ }^{2}$ \& María Cecilia Piedra Rivas. ${ }^{3}$

\begin{abstract}
Abstrac.
Introduction. Agriculture is one of the most important economic sectors for Latin American countries. Government policies should be aimed at strengthening this agricultural sector for the food security of the countries, with the appropriate measures for the protection of the environment. The role of agriculture in the economy reaffirms the thesis that it is important not only because it constitutes the way of life of millions of people in the Ecuadorian countryside, but also because it is a strategic sector that contributes directly to rural territorial development, and therefore, to the economic and social development of the country. Revaluing the peasant producer and repositioning agriculture and rural life is one of the main challenges of continuous policy evaluation. The Inter-American Development Bank (IDB) has created a platform with agricultural data from Latin American countries - Agrimonitor. Objective. The problem addressed in this research is that the government of Ecuador and its exporters of agricultural products should use this platform to better direct their production, the objective is to make this tool and its management known to take advantage of the country's agricultural sector. Methodology. The research design is qualitative with the method of observation and reading of documents issued by the IDB and works related to the issue of strengthening

\footnotetext{
${ }^{1}$ Economista, MBA, investigador independiente. Azuay-Ecuador beteml@hotmail.com ORCID: 00000001-9264-2251

2 Ing Agropecuario, MSc Desarrollo Local, Azuay - Ecuador rcalvear77@ gmail.com ORCID: 0000-00019270-714X

${ }^{3}$ Ing Agrónomo, MSc Desarrollo Local, Azuay- Ecuador cecilia.piedra@yahoo.es ORCID:0000-00032813-274
} 
agricultural production in the country. Results and conclusions. It is concluded that the Agrimonitor tool could be of great importance for state decisions in the agricultural field.

Key words: agriculture, rural development, agriculture, politics

\section{Resumen.}

Introducción. La agricultura es uno de los sectores económicos de mayor relevancia para los países de Latinoamérica, las políticas gubernamentales deben ir dirigidas en afianzar este sector agropecuario para la seguridad alimentaria de los países, con las debidas medidas de la protección del medio ambiente. El rol que cumple la agricultura en la dinamización económica de todos los países, es muy importante, y en el Ecuador no sólo porque constituye la forma de vida de millones de personas del campo ecuatoriano, sino también porque es un sector estratégico que contribuye directamente al desarrollo territorial rural, y por ende, al desarrollo económico y social del país. Revalorizar al productor campesino y reposicionar la agricultura y la vida rural es uno de los principales desafíos de continua evaluación de políticas. El Banco Interamericano de Desarrollo (BID) ha creado una plataforma con datos agrícolas de los países de Latinoamérica Agrimonitor -. Objetivo. El problema tratado en esta investigación es que el gobierno del Ecuador y sus exportadores de productos agrícolas deberían utilizar esta plataforma para poder dirigir de mejor manera su producción, el objetivo es hacer conocer esta herramienta y su manejo para el aprovechamiento del sector agropecuario del país. Metodología. El diseño de la investigación es cualitativo con el método de observación y lectura de documentos emitidos por el BID y obras relacionadas al tema del fortalecimiento de la producción agrícola en el país. Resultados y conclusiones. Se concluye que la herramienta Agrimonitor podría ser de gran importancia para las decisiones estatales en el ámbito agropecuario.

Palabras Clave: agricultura, desarrollo rural, agropecuaria, políticas

\section{Introducción.}

El sector agropecuario aporta en gran medida a la economía y a la sociedad, muchas veces una contribución invisibilizada. Este sector, según Reinoso (2013) aporta al menos con la quinta parte de la producción de bienes y servicios del país, en sentido ampliado; produce el $95 \%$ de los bienes alimenticios que se consumen internamente; ocupa el $62 \%$ de la población económicamente activa rural (la mayoría de esta población genera su propio empleo); el $46 \%$ de su producción es fuente de insumos para otras actividades productivas (consumo intermedio); aporta a la liquidez monetaria, pues el $40 \%$ de las divisas que ingresaron al país por exportaciones en el presente siglo en promedio anual provienen de este sector. Dado su bajo componente de importaciones, incide en el saldo positivo de la balanza comercial agropecuaria, contribuye a equilibrar el saldo negativo en la balanza 
comercial global (industrial) y ayuda a garantizar el puente intergeneracional de la sociedad a través de la seguridad y soberanía alimentaria, entre otros aportes.

La importancia del sector agropecuario en el Ecuador se debe principalmente a tres aspectos; primero, por su representatividad en el PIB, segundo, por constituir una fuente de divisas a través de la exportación tanto de productos tradicionales y no tradicionales. Finalmente, por constituir la base de la política de soberanía alimentaria promovida por la actual Constitución en la que se menciona que la soberanía alimentaria constituye un objetivo estratégico y una obligación del Estado para garantizar que las personas, comunidades, pueblos y nacionalidades alcancen la autosuficiencia de alimentos sanos y culturalmente apropiado de forma permanente. (Reinoso, 2013)

De acuerdo a cifras del INEC (2021) por medio la Encuesta de Superficie y Producción Agropecuaria Continúa - ESPAC- es la principal fuente de información oficial sobre el sector agropecuario, con un marco de muestreo adoptado de acuerdo a las recomendaciones de la Organización de las Naciones Unidas para la Alimentación y la Agricultura (FAO, por sus siglas en inglés). Se recopiló la información relacionada a la superficie plantada y cosechada de 52 productos agrícolas específicos, información sobre ganados, aves y empleo del sector agropecuario.

Tabla 1

Superficie con usos agropecuario en el Ecuador

\begin{tabular}{lrr}
\hline \multicolumn{1}{c}{ Superficie con uso agropecuario } & \multicolumn{1}{c}{$\mathbf{2 0 1 8}^{*}$} & \multicolumn{1}{c}{$\mathbf{2 0 1 9}^{*}$} \\
\hline Cultivos Permanentes & 1.385 .805 & 1.439 .504 \\
Cultivos Transitorios y Barbecho & 799.494 & 769.708 \\
Pastos Cultivados & 2.379 .042 & 1.985 .494 \\
Pastos Naturales & 715.273 & 915.843 \\
\hline
\end{tabular}

Fuente: (INEC, 2021)

Los principales productos del sector agrícola en el Ecuador son la caña de azúcar, banano, palma africana, café, cacao, arroz, papa, maíz maduro. En ganado predomina el ganado vacuno con su producción lechera y de carne; el avícola que contempla también la producción de huevos según dato del INEC (2021) que sirven para el consumo interno y exportación. Las exportaciones de los productos agrícolas tradicionales y no tradicionales significan un $8 \%$ del PIB según datos del Ministerio de Agricultura y Ganadería del Ecuador (2021), que es un porcentaje interesante para que el Gobierno Nacional de vuelta su mirada al sector agrícola como fuente para desarrollar alternativas económicas y fuentes de empleo a nivel nacional.

Por otro lado, según datos del MAG (2021) se encontró que luego de medio siglo de intervenciones estatales, los problemas estructurales del sector agropecuario ecuatoriano prevalecen; es más, hoy se suman nuevos desafíos. Sesenta años después de la primera reforma agraria, los pequeños campesinos siguen controlando casi la misma superficie de tierra: 6,7\% en 1954 frente a 6,5 en 2013. El coeficiente de Gini, varió muy poco: en 1954 
éste era de 0,87, para el 2013 registra 0,76. Es decir, la redistribución se dio principalmente por la fragmentación de la gran propiedad hacia unidades productivas de tamaño intermedio (entre 20 y menos de 100 ha), mientras en 1954 la mediana propiedad controlaba el 17,8\% de la superficie nacional, al 2013, controla el 43,2\%.

La falta de políticas públicas diferenciadas territorialmente implica desconocer la marcada heterogeneidad territorial en los sistemas de producción agropecuaria y ha llevado a la ausencia de una agricultura más incluyente y capaz de aprovechar plenamente el potencial productivo del país en concordancia con las diversas condiciones biofísicas, agroecológicas, así como los desiguales sistemas de producción y los distintos sistemas de organización social, económica y cultural de los productores. Todo ello ha limitado el funcionamiento de estrategias de alto impacto sobre la agricultura y sus potenciales contribuciones para salir de la pobreza y contribuir al desarrollo; sumado a eso en Latinoamérica y Ecuador los continuos cambios de gobierno y políticas a largo plazo.

Para que los países de Latinoamérica tengan información sobre la producción agrícola de los países de la región el BID ha desarrollado una herramienta llamada Agrimonitor en donde se puede encontrar desde indicadores agropecuarios de países hasta información relacionada con seguridad alimentaria y cambio climático. Esta herramienta tiene como enfoque de los Estimados de Apoyo al Productor (siglas PSE en inglés) sigue el marco de análisis de la Organización para la Cooperación y el Desarrollo Económicos -OCDEcon el fin de permitir la comparación de las políticas agropecuarias entre países. La metodología* es utilizada actualmente por 39 miembros de la OCDE y las economías emergentes para monitorear las políticas agropecuarias. La comparabilidad de estos indicadores de política y su potencial para la modelación hacen que la base de datos sea particularmente valiosa para América Latina y el Caribe. El PSE y los indicadores relacionados se basan en la interacción de oferta, demanda y transacciones entre los agricultores, los consumidores y los contribuyentes, con el fin de medir los incentivos o transferencias y desincentivos de política pública para el sector agropecuario. La página web de (Agrimonitor, 2021) es http://agrimonitor.iadb.org/es/

El problema tratado en la investigación es que las autoridades del sector agropecuario en el Ecuador no están utilizando la herramienta Agrimonitor para poder tomar decisiones en su producción; el objetivo es que los agricultores y exportadores agrícolas del país tengan una visión de lo que sucede internamente y dentro del mercado de Latinoamérica y el Caribe, para que puedan tener juicios de valor en los productos a producir, las necesidades de toda la región y como minimizar el impacto ambiental. La metodología utilizada fue la cualitativa con métodos de lectura y revisión de los documentos del BID y de autores que tratan sobre la industria agropecuaria en el Ecuador, finalmente la conclusión es que la herramienta debe ser facilitada y explicada por el Gobierno Nacional por medio del Ministerio de Agricultura y Ganadería a las diferentes ramas de la economía agrícola.

A continuación, en el marco teórico con el aporte de algunas obras se resalta la importancia de la agricultura en el Ecuador, la seguridad alimentaria, la protección del 
medio ambiente, y algunos temas relacionados a la investigación; se da a conocer la metodología utilizada; posterior un análisis de los resultados y discusión y se termina con las conclusiones del artículo.

\section{Marco Teórico.}

Diversos encuentros mundiales y regionales sobre desarrollo han evidenciado los retos futuros en materia de bienestar, sustentabilidad y equidad; han remarcado la importancia de reducir la pobreza, erradicar el hambre, mejorar la nutrición, proteger los ecosistemas y desarrollar economías incluyentes, entre otros. Dichos aspectos están relacionados con la adaptación de la agricultura al cambio climático, la inclusión rural, la seguridad alimentaria y nutricional y el manejo integral y sustentable de recursos naturales (IICA, Informe anual del 2019. Ecuador: logros significativos, 2019). Estos son aspectos clave en un diseño de política agropecuaria.

Recientes análisis de la Comisión Económica para América Latina y el Caribe, (CEPAL), la Organización de las Naciones Unidas para la Alimentación y la Agricultura, FAO, el Instituto Interamericano de Cooperación para la Agricultura, IICA, y otros señalan que el intercambio global de América Latina y el Caribe enfrentará un escenario de estancamiento en algunos de sus principales mercados, como Europa y Estados Unidos. En cambio, en el mercado internacional de bienes agrícolas, la competitividad de América Latina y el Caribe ha crecido sostenidamente por más de una década. Ecuador hace parte de esa tendencia. La región, altamente especializada en la exportación de productos agrícolas, mantiene un buen dinamismo y se posiciona mejor que otras. Se estima que la demanda internacional de bienes agrícolas continuará creciendo, mientras que la oferta de alimentos y materias primas agrícolas no crecerá al mismo ritmo (IICA, 2012).

En el estudio reciente de la CEPAL (2018) "Perspectivas de la agricultura y del desarrollo rural en las Américas", elaborado por CEPAL, FAO, IICA se advierte que dadas las limitaciones en recursos naturales, las presiones ambientales, el cambio climático y la mayor volatilidad de precios, el principal desafío que enfrenta el sector agrícola es aumentar la productividad en forma amigable con el ambiente. También concluyen que las condiciones climáticas extremas, los riesgos de un posible colapso del euro, un posible estancamiento fiscal de los EE.UU. y la ralentización de las economías emergentes, entre otros, apuntan a un ambiente de mayor incertidumbre y volatilidad en los precios internacionales agrícolas.

En el último cuarto de siglo, se han identificado rasgos comunes que han dominado la política agropecuaria latinoamericana. Esta se ha concentrado en temas de innovación tecnológica, sanidad-inocuidad, riego-drenaje y financiamiento, sin desarrollar mayores esfuerzos en cuanto al modelo productivo vigente. Según PIADAL (2013) Durante su implementación han dominado las políticas macroeconómicas de incidencia directa en el sector agropecuario; las de mayor relevancia son las cambiarias, monetarias, fiscales, tributarias y comerciales. 
El análisis de PIADAL (2013) concluye que las políticas para el sector agropecuario en las últimas décadas en América Latina no han sido suficientemente efectivas para impulsar un desarrollo sólido y equilibrado. Por lo tanto, urge desarrollar nuevas estrategias dirigidas a lograr un desarrollo más armónico en la región. En este sentido, CEPAL, FAO e IICA sostienen que el desarrollo de la agricultura dependerá de la apropiada implementación de un conjunto integrado de políticas sectoriales y extra sectoriales, adecuadas a la realidad de cada país. Según IICA (2014) identifica tres ejes fundamentales en la formulación de las futuras políticas agropecuarias: el primero apunta a aumentar los niveles de productividad y competitividad, para lo cual se requiere generar, adaptar y validar tecnologías, además de desarrollar procesos innovadores que permitan aumentar los rendimientos y la eficiencia en el uso sostenible de los recursos naturales, en especial del agua y el suelo.

El segundo eje en las recomendaciones de política agropecuaria para la región es apoyar a la agricultura de pequeña escala y a la agricultura familiar. Según IICA (2019), el grado de vulnerabilidad de los pequeños productores rurales a la pobreza, y en consecuencia al hambre, se puede reducir brindando apoyo a este grupo de productores. El IICA (2019) sostiene que una agricultura promotora del crecimiento económico orientado hacia el bienestar rural solo será posible si se logra que los productores y los habitantes rurales gocen de mayor inclusión a los beneficios del desarrollo, la diversificación y la agregación de valor.

Finalmente, el tercer aspecto a considerar en los diseños de política es considerar explícitamente a las mujeres, "las mujeres rurales constituyen una de las fuerzas motrices de la economía de los territorios y son corresponsables del desarrollo, la estabilidad y la supervivencia de sus familias. Como productoras de alimentos asociadas a la agricultura familiar y dado el rol que desempeñan en la toma de decisiones sobre el uso y la distribución de los ingresos familiares" (IICA, 2014).

En suma, las políticas agropecuarias aplicadas en la región en las últimas décadas, no han logrado innovar los modelos de producción agrícola vigentes desde hace más de tres décadas atrás; ello sin dejar de reconocer los avances que excepcionalmente se experimentaron en algunos países latinoamericanos, en términos de mejoría en la situación de pequeños agricultores familiares, cuyos esfuerzos al parecer lograron reducir la pobreza rural. Por ello se requiere introducir transformaciones importantes a las políticas agropecuarias; la experiencia acumulada en varios países latinoamericanos en materia agropecuaria constituye sin duda un referente importante para no cometer los mismos errores u omisiones del pasado, o para aprender lo positivo de aquellos ejemplos.

Aguilar (2005) contribuye en una de sus investigaciones que no es posible generar un crecimiento económico y un desarrollo sostenible en el continente latinoamericano, sin invertir en el sector agropecuario. El fortalecimiento del sector agrícola, en Latinoamérica, es de gran importancia para la región debido a su relevancia, no solo en la estabilidad macroeconómica, sino también por su papel en la generación de empleo en los sectores rurales donde se concentra la pobreza. Las nuevas ventanas de mercado, 
debido a los tratados de libre comercio en la región, buscan fortalecer todos los sectores de la economía, entre esos la agricultura, de la misma manera, se busca una competitividad generada por la innovación tecnológica, productividad, entre otras. Esto no deja de ser un reto para los países de la región, que aparte de especializarse en los productos en los cuales tienen ventajas comparativas, deben crear estrategias para fortalecer el sector rural, y aprovechar esta oportunidad que genera la apertura de mercados.

Los gobiernos latinoamericanos han tratado de promover la seguridad alimentaria en sus respectivos países, es así que el Comité Científico de la ELCSA (2012) en Latinoamérica en la lucha contra la inseguridad alimentaria, la medición adecuada de este fenómeno se ha constituido en un componente esencial de los esfuerzos por superarlo. Diversas agencias de desarrollo, gubernamentales y no gubernamentales, ministerios y secretarías, programas de asistencia locales y nacionales, e instancias académicas abocadas al estudio de la inseguridad alimentaria, sus causas y consecuencias, requieren sistemas de evaluación y monitoreo que hayan comprobado su validez y confiabilidad. Solo con instrumentos adecuados de medición será posible comprender mejor las causas subyacentes a la inseguridad alimentaria, identificar y localizar las poblaciones en mayor riesgo, determinar mecanismos efectivos para atacar el problema y evaluar el impacto de las diversas intervenciones que se propongan. Para la medición de la inseguridad alimentaria se han usado tradicionalmente métodos basados en indicadores económicos de producción y disponibilidad de alimentos a nivel nacional y regional. Desafortunadamente, estos son métodos caros, que dependen en buena medida de la capacidad de los países y gobiernos de generar los datos necesarios de manera regular, usando técnicas estandarizadas, y no proveen información que refleje el acceso de los hogares a los alimentos disponibles.

Dado que la seguridad alimentaria se basa en la capacidad de acceso a los alimentos, la medición de la inseguridad alimentaria debería centrarse asimismo en el acceso y no solamente en algunas de sus causas (por ejemplo la falta de disponibilidad de alimentos o los niveles de pobreza) o solo en algunos de sus efectos (por ejemplo la desnutrición crónica en niños, la baja diversidad de la dieta). Alternativamente, el acceso puede medirse en forma directa por medio de la experiencia de los hogares cuando enfrentan diversos niveles de inseguridad alimentaria. Adicionalmente, las escalas basadas en la experiencia de los hogares permiten capturar las dimensiones subyacentes de la inseguridad alimentaria (la dimensión psicológica, la calidad y cantidad de los alimentos, la presencia de hambre no satisfecha).

Es importante impulsar la agricultura como medio para potenciar las economías, pero es de suma importancia también cuidar el medio ambiente y tratar que el sector agrícola no afecte los recursos naturales de un país, como lo expone el autor Bartra (2002) el medio ambiente provee el entorno necesario para la vida humana, flora y fauna. Los recursos naturales, patrimonio de la nación, constituyen los elementos materiales necesarios para satisfacer los requerimientos de alimentación, vestido, vivienda, energía y demás 
productos de la población, pero también deben de garantizar el bienestar de las generaciones futuras.

Los autores Carrión y Herrera (2012) se manifiestan sobre la importancia de la agricultura en la economía ecuatoriana. Se parte con una reflexión sobre el peso del sector agrícola desde 1980, es decir, desde la década en que la caída de las exportaciones petroleras y las medidas de ajuste devolvieron al sector agroexportador su relevancia en la escena económica nacional, el petróleo y las agroexportaciones resultan indicativos del modelo económico que históricamente se sostiene sobre la exportación de bienes primarios. Según MAG (2015) algunos productos exportables ecuatorianos destacan en el mercado mundial. Ecuador es el mayor exportador de banano del mundo, el segundo exportador de camarón y atún, y el tercer exportador de flores. Es decir, aún hay oportunidades para aprovechar más estos productos tradicionales. Luego, haciendo un análisis comparativo del crecimiento del sector agropecuario frente a otros sectores de la economía, se introduce el panorama actual, mostrando el decrecimiento de la producción típicamente campesina frente a la agroindustria, que se revela contraria a los avances constitucionales en torno a la soberanía alimentaria. Diversificar la producción agrícola más allá de los commodities, fortaleciendo los productos agropecuarios andinos, tradicionales $\mathrm{y}$ orgánicos, la horticultura, la fruticultura no tradicional, los granos andinos, además de la merluza y la cobia, se visualiza como una gran oportunidad para el país. Se prevé que los precios de estos últimos crecerán.

De acuerdo a MAG (2015) desde mediados de los años sesenta del siglo XX hasta la actualidad, la política agropecuaria ecuatoriana ha transitado por distintos direccionamientos, enfoques y modelos de intervención de la política pública, cuyos mecanismos han conducido a cambios en la estructura agraria y de poder importantes.

El Agrimonitor es una herramienta desarrollada por el BID en donde se puede encontrar desde indicadores agropecuarios de países hasta información relacionada con seguridad alimentaria y cambio climático países latinoamericanos.

\section{Metodología.}

Se utilizó el método investigación exploratoria cualitativa, que se empleó para estudiar una herramienta agrícola que aún no está claramente definido en Latinoamérica; por lo que se ha tomado como base documentos del BID en la cual se explica la utilización de esta herramienta.

Según Mejía (2004) la investigación cualitativa es el procedimiento metodológico que utiliza palabras, textos, discursos, dibujos, gráficos e imágenes para comprender la vida social por medio de significados y desde una perspectiva holística, pues se trata de entender el conjunto de cualidades interrelacionadas que caracterizan a un determinado fenómeno, utiliza descripciones detalladas de hechos, citas directas del habla de las personas y extractos de pasajes enteros de documentos para construir un conocimiento de la realidad social, en un proceso de conquista construcción-comprobación teórica. 


\section{Resultados y Discusión.}

El BID ha desarrollado una herramienta mediante una página para apoyar al sistema agrícola de los países latinoamericanos, a continuación, se da a conocer el manejo de la web y que datos se pueden encontrar en ella. La información fue extraída de la página web de Agrimonitor (2021) que se le encuentra en el siguiente link: http://agrimonitor.iadb.org/es/

A continuación, se da a conocer los siguientes pasos para poder explorar la información de la web de Agrimonitor.

Paso 1: La página de inicio de Agrimonitor.

\section{Figura 1}

Página de inicio de Agrimonitor

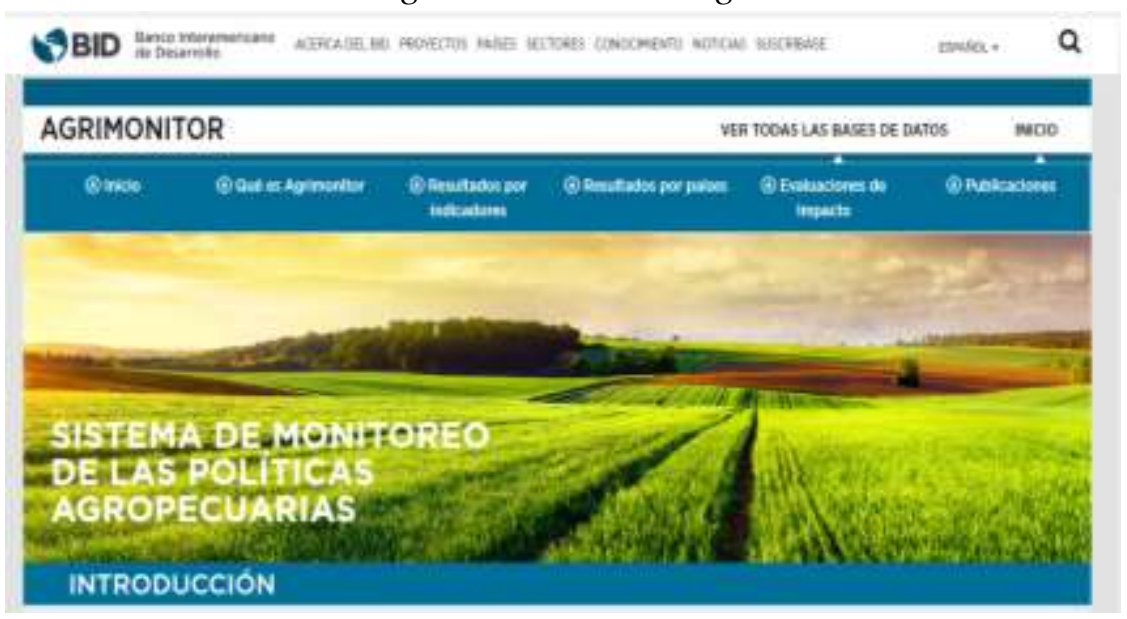

Fuente: (Agrimonitor, 2021)

En Agrimonitor se puede encontrar desde indicadores agropecuarios de países hasta información relacionada con seguridad alimentaria y cambio climático.

Se ira revisando cada una de la subpestañas de la página web que consta de 5 elementos, que se detallan a continuación:

\section{Figura 2}

Subpestañas del Agrimonitor

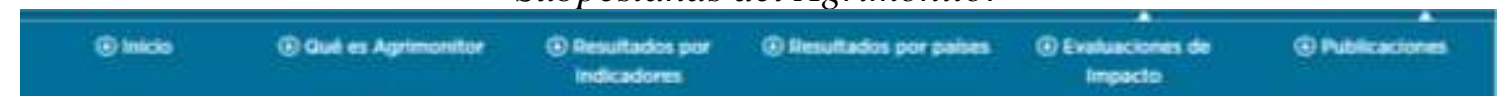

Fuente: (Agrimonitor, 2021)

Paso 2: ¿Qué es Agrimonitor?

La sección "Qué es Agrimonitor" contiene dos subpestañas y su objetivo es que el lector tenga una idea sobre el significado de Agrimonitor y sus orígenes.

Enfoque, estructura y conclusiones: en esta página se puede observar una descripción general sobre la metodología de Estimados de Apoyo al Productor -EAP-. 
Figura 3

Enfoque, estructura y conclusiones del Agrimonitor

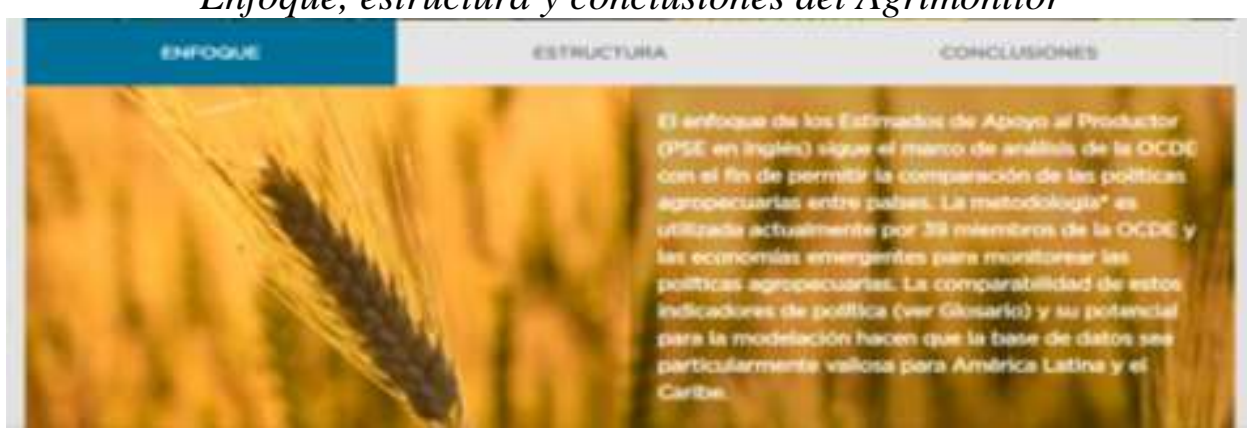

Fuente: (Agrimonitor, 2021)

Glosario: esta página te permite encontrar, en un solo lugar, los significados de las siglas de los indicadores claves relacionados con la Metodología EAP.

\section{Figura 4}

Glosario del Agrimonitor

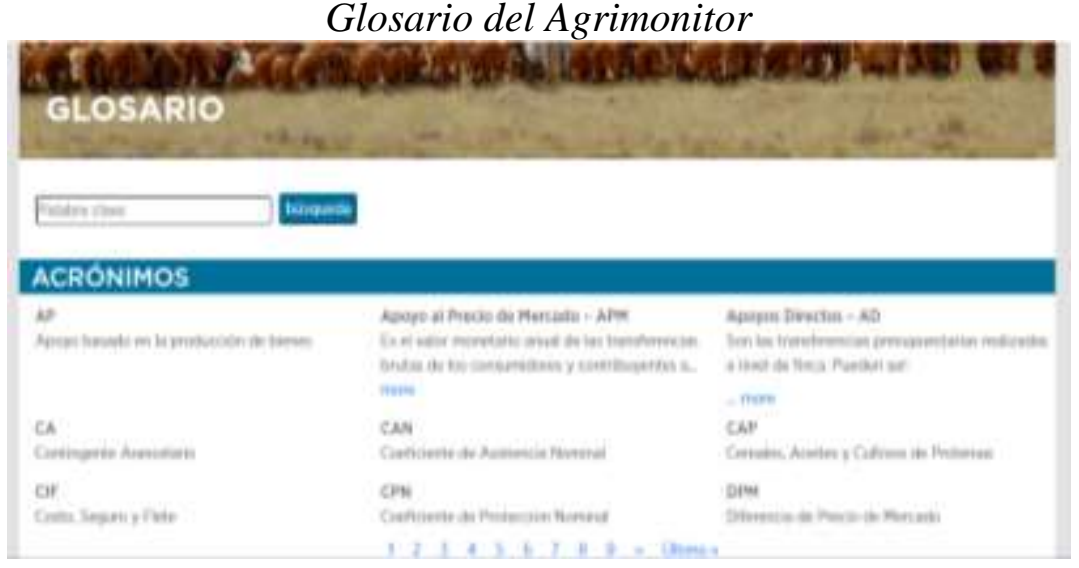

Fuente: (Agrimonitor, 2021)

Paso 3: Resultados por indicadores.

En la pestaña "Resultados por indicadores", se podrá encontrar, como lo dice su nombre, datos generales sobre los siguientes cuatro indicadores que se ve en la imagen y realizar una comparación rápida entre países.

\section{Figura 5}

\section{Resultados por indicadores}

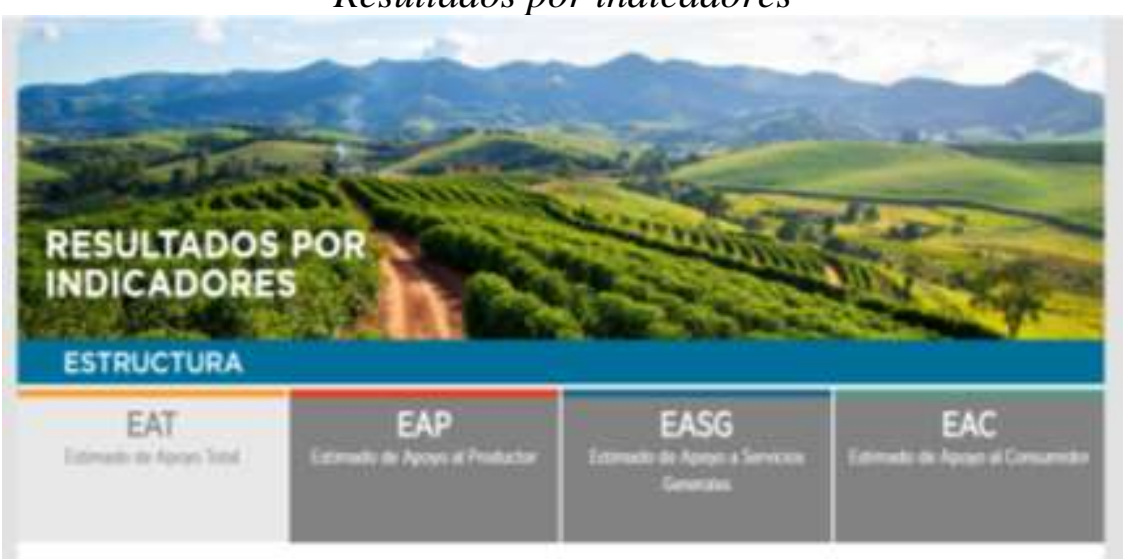

Fuente: (Agrimonitor, 2021) 
EAT (Estimado de Apoyo Total): EAT o TSE en inglés refleja el apoyo público total al sector agropecuario. Este indicador incluye todos los efectos de las políticas públicas que afectan de manera diferencial al sector, sean estas de apoyo (por ejemplo, mediante subsidios), o de penalización (por ejemplo, mediante impuestos). El EAT\% indica el apoyo al sector como porcentaje del Producto Interno Bruto (PIB) de un país.

Tabla 2

EAT 2019 en Latinoamérica

\begin{tabular}{ccc}
\hline PAIS & EN MILLONES USD & EN BILLONES USD \\
\hline Unión Europea & & 113,32 \\
México & & 6,16 \\
Estados Unidos & 421,72 & 100,08 \\
Costa Rica & 818,17 & \\
Colombia & & \\
Canadá & & 4,94 \\
Argentina & & $-11,49$ \\
\hline
\end{tabular}

Fuente: (Agrimonitor, 2021)

EAP (Estimado de Apoyo al Productor): EAP o PSE en inglés explica el nivel de apoyo que los productores agropecuarios reciben mediante políticas agropecuarias a nivel de finca. Es el valor monetario anual de las transferencias brutas de consumidores y contribuyentes a los productores agrícolas, medido a nivel de la granja, a partir de medidas de política que respaldan la agricultura, independientemente de su naturaleza, objetivos o impactos en la producción o los ingresos agrícolas. El EAP\% refleja este apoyo como porcentaje de los ingresos brutos de la finca.

Tabla 3

EAP 2019 en Latinoamérica

\begin{tabular}{cc}
\hline PAIS & EN BILLONES USD \\
\hline Estados Unidos & 101,25 \\
Chile & 5,34 \\
Argentina & 48,93 \\
Costa Rica & 3,53 \\
México & 4,94 \\
Brasil & 11,49 \\
\hline
\end{tabular}

Fuente: (Agrimonitor, 2021)

EASG (Estimado de Apoyo a Servicios Generales): EASG o GSSE en inglés mide el apoyo que se brinda a los productores agropecuarios (como sector y no individualmente) a través de servicios generales. Es el valor monetario anual de las transferencias brutas a servicios generales provistos colectivamente a los productores agrícolas (como investigación, desarrollo, capacitación, inspección, comercialización y promoción), a partir de medidas de política que apoyan a la agricultura independientemente de su naturaleza, objetivos e impacto en la producción de la finca, ingreso o consumo. El GSSE no incluye ninguna transferencia a productores individuales. El EASG\% indica el porcentaje de este apoyo respecto al apoyo total a los productores agropecuarios. 
Tabla 4

EASG 2019 en Latinoamérica

\begin{tabular}{ccc}
\hline PAIS & EN MILLONES USD & EN BILLONES USD \\
\hline Unión Europea & & 11,69 \\
México & 536,78 & 10,96 \\
Estados Unidos & & \\
Colombia & 454,84 & 1,42 \\
Canadá & & 2,19 \\
Brasil & & \\
Argentina & 244,84 & \\
\hline
\end{tabular}

Fuente: (Agrimonitor, 2021)

EAC (Estimado de Apoyo al Consumidor): EAC o CSE en inglés indica cómo las políticas agropecuarias afectan al consumidor de productos agropecuarios. Es el valor monetario anual de las transferencias brutas de (hacia) consumidores, medido a nivel de finca, y que surge de medidas de política que respaldan a la agricultura, independientemente de su naturaleza, objetivos o impactos en el consumo de productos agrícolas. El EAC\% refleja el porcentaje del EAC respecto al costo de la canasta de productos agropecuarios, es decir el EAC como parte del gasto de consumo, medido a nivel de finca y neto de las transferencias de los contribuyentes a los consumidores.

Tabla 5

EAC 2019 en Latinoamérica

\begin{tabular}{ccc}
\hline PAIS & EN MILLONES USD & EN BILLONES USD \\
\hline Unión Europea & & $-16,53$ \\
México & & 26,22 \\
Estados Unidos & 0 & \\
Costa Rica & $-312,43$ & \\
Colombia & 0 & $-2,40$ \\
Chile & & \\
Canadá & 879,18 & 8,22 \\
Brasil & & \\
Argentina & &
\end{tabular}

Fuente: (Agrimonitor, 2021)

Paso 4: Resultados por países.

La pestaña "Resultados por países", contiene dos subpestañas; indicadores por países y comparar resultados.

Indicadores por países: en esta sección, se puede generar gráficos con información detallada y específica sobre indicadores agropecuarios por país. 


\section{Figura 6}

Indicadores por países

\begin{tabular}{|c|c|c|c|}
\hline Indicadores por paises & & & \\
\hline Argentina & Colombia & Haití & Surinam \\
\hline Bahamas & Costa Rica & Honduras & Trinidad y Tobago \\
\hline Barbados & $\overline{\text { República Dominicana }}$ & Jamaica & Estados Unidos \\
\hline Belice & Ecuador & México & Uruguay \\
\hline Bolivia & El Salvador & Nicaragua & Venezuela \\
\hline$\overline{\text { Brasil }}$ & Unión Europea & Panamá & \\
\hline Canadá & Guatemala & Paraguay & \\
\hline Chile & Guyana & Perú & \\
\hline
\end{tabular}

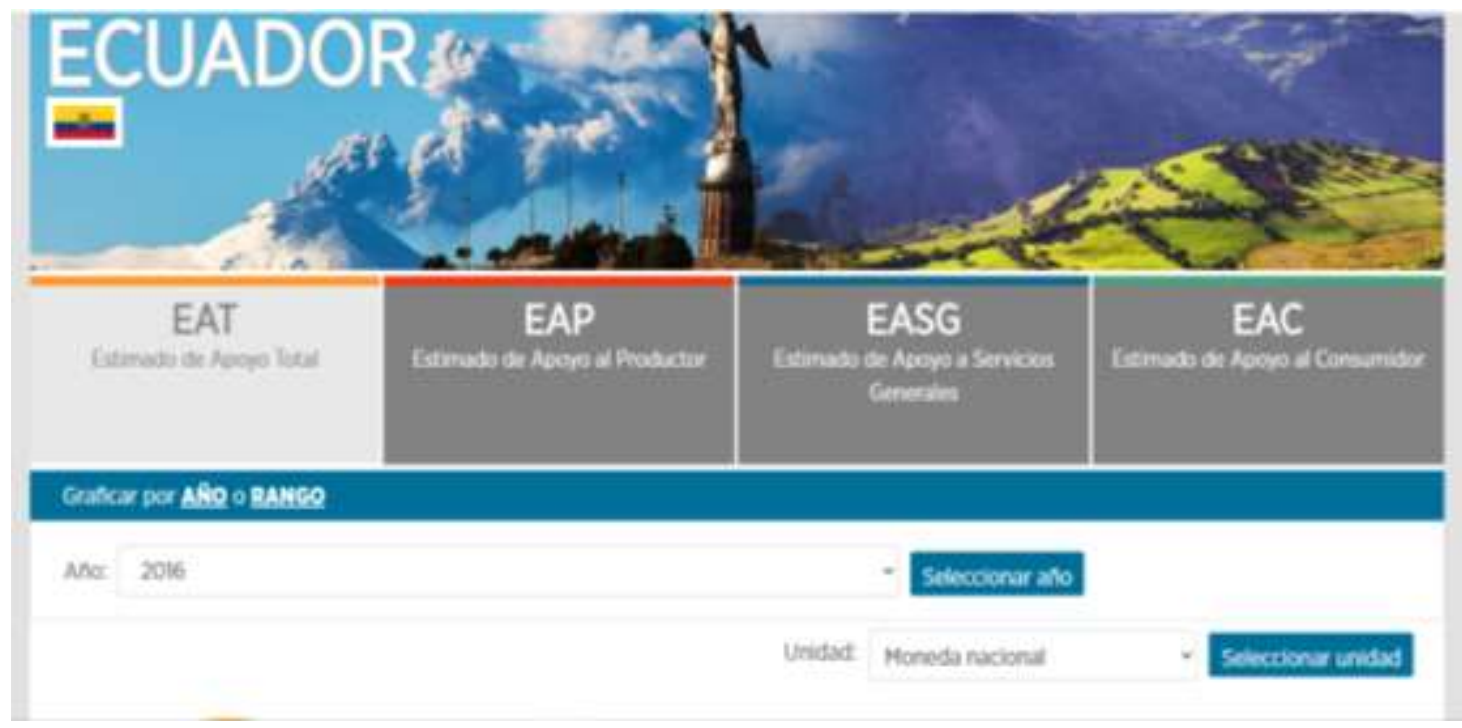

Fuente:_(Agrimonitor, 2021)

El EAT en el Ecuador en el año 2016 es de 1.12 billones de dólares; expresado en los siguientes componentes:

Tabla 6

Estimado de apoyo total Ecuador 2016

\begin{tabular}{ccc}
\hline CSE & GSSE & PSE \\
\hline$-795,19$ Millones & 100,31 Millones & 1,01 billones \\
\hline
\end{tabular}

Fuente: (Agrimonitor, 2021)

Comparar resultados: en esta sección, se puede generar gráficos para comparar información detallada y específica sobre indicadores agropecuarios entre países. 
Figura 7

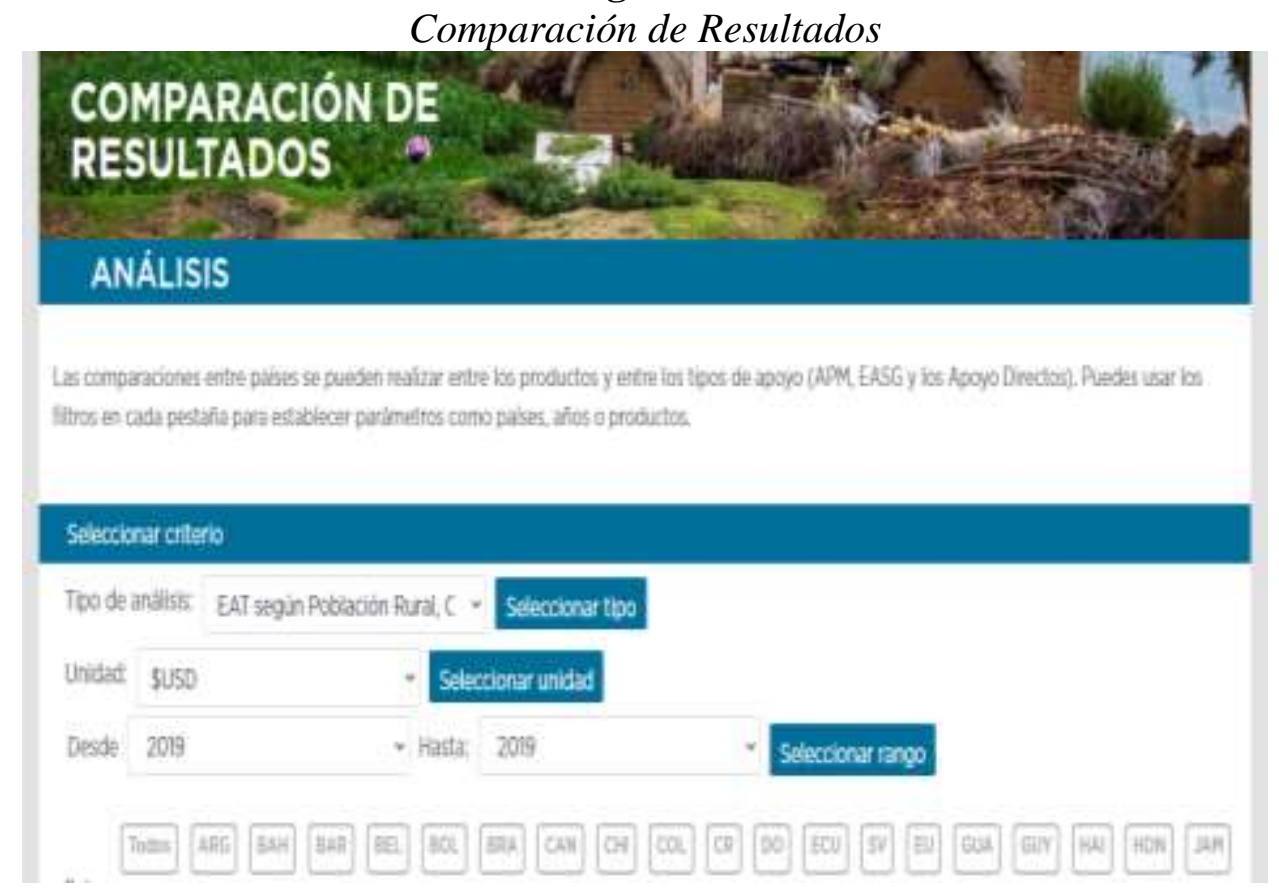

Fuente: (Agrimonitor, 2021)

Las comparaciones entre países se pueden realizar entre los productos y entre los tipos de apoyo (APM, EASG y los Apoyo Directos). Se puede usar los filtros en cada pestaña para establecer parámetros como países, años o productos.

\section{Paso 5: Evaluaciones de Impacto.}

La pestaña "Evaluaciones de Impacto": en esta sección se puede encontrar publicaciones del BID asociadas a evaluaciones de impacto de países asociados a Agrimonitor. Por el momento, la página solo refleja estudios para algunos países, pero se sigue poblando con más información.

Se coloca el mouse sobre uno de los países marcados en azul para encontrar las publicaciones asociadas a ese país.

La evaluación de impacto es un tipo de análisis que complementa los estimativos del PSE. Mientras que el PSE mide la composición del apoyo público en agricultura, las evaluaciones de impacto miden la efectividad de estos apoyos (políticas/programas). Específicamente, las evaluaciones de impacto se enfocan en determinar el efecto causal de una política o programa en el grupo de beneficiarios. Es decir, este tipo de análisis mide que hubiera pasado a los beneficiarios en la ausencia el programa. Para lograr este fin se utilizan herramientas que permiten identificar un grupo de control comparable ya sea a través de metodologías experimentales o cuasi-experimentales. Este grupo de control está compuesto por unidades que no participaron en el programa pero que son comparables al grupo de beneficiarios. Los resultados de un estudio de impacto pueden ser utilizados para complementar los análisis costo-beneficio a fin de definir la viabilidad económica de diferentes políticas/programas. También sirven para extrapolar posibles 
beneficios a un grupo poblacional más grande y por ende, evaluar la viabilidad de extender una política/programa hacia otros beneficiarios o territorios.

\section{Paso 6: Publicaciones.}

La pestaña "Publicaciones", aquí se puede encontrar interesantes publicaciones del BID acerca de temáticas agropecuarias, como informes de países, papers, manuales, entre otros. También, puedes utilizar la barra de búsqueda para encontrar una publicación de un tema específico de tu interés relacionado con políticas agropecuarias:

\section{Figura 8}

\section{Publicaciones}

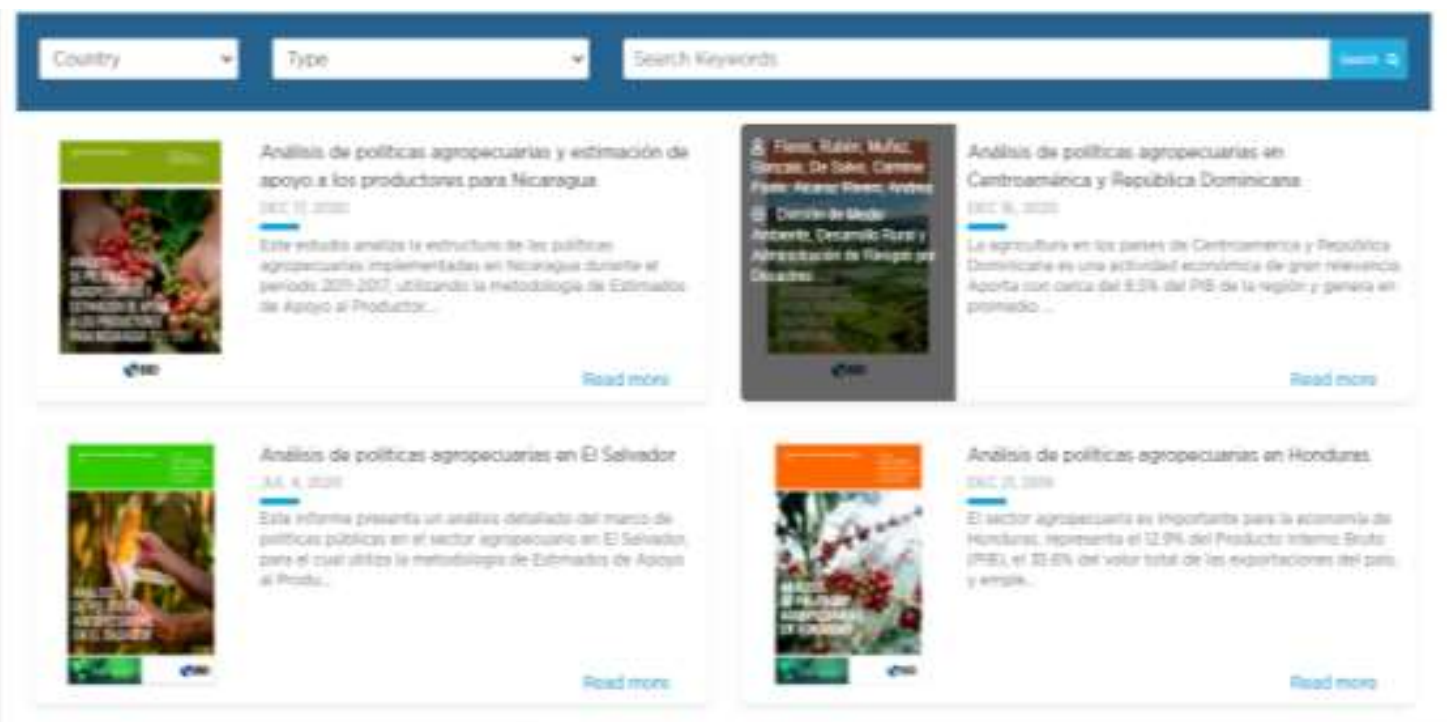

Fuente: (Agrimonitor, 2021)

Como se puede observar los datos de la plataforma del Agrimonitor pueden ser de gran importancia para el sector agrícola del Ecuador, lamentablemente los encargados por parte del gobierno de entregar la información no lo hacen de forma completa y lo están haciendo de forma atrasada, como se revisa en los ejemplos el Ecuador tiene ingresado datos al 2016, por lo que no se pueden comparar con el resto de países que tiene ingresado su información al 2019.

Para cerrar el análisis de los resultados y la discusión; se da a conocer este enunciado que es muy importante para la economía agropecuaria en el Ecuador; la agricultura requiere de apoyo sustancial, puesto que la calidad de vida de la mayoría de los pequeños agricultores es precaria. A través de las instituciones estatales focalizadas en el sector agrícola y con la ayuda de empresas privadas e incluso organizaciones no gubernamentales, se debería llevar a cabo un plan de cadenas productivas que apoyen a los sectores rurales más pequeños para que se conviertan en los principales proveedores de la industria, puesto que el principal problema que enfrentan los agricultores, es la comercialización. Para lograr esto los productores necesitan mejorar su capacidad productiva y la calidad de sus productos, para lo que programas apoyados por los 
diferentes sectores deben enfocarse en que haya mejor acceso a los recursos tradicionales (tierra, agua, educación) y no tradicionales (insumos mejorados, asistencia técnica, infraestructura); además, se deberían motivar asociaciones colectivas en las diferentes zonas, para que los productores trabajen en conjunto y alcancen economías de escala, poder de negociación (incentivos de precios), entre otros. A nivel agrícola, también se pueden lograr aglomeraciones o clusters cooperativos, que aunque los productores compitan entre ellos, pueden unirse para desarrollar el cluster y hacerlo más competitivo y al mismo tiempo para lograr que se genere otro tipo de empleo en la zona rural. Los proyectos de microcrédito han demostrado ser efectivos en la reducción de pobreza a este nivel en muchos otros países, por lo que se debería fortalecer programas de este tipo. Deben apoyarse los cultivos de productos que sostengan la alimentación interna. En general, lo que se ha invertido en agricultura es casi nulo en comparación con los otros sectores, por lo que la inversión es urgente (ANDREALIZ’S, 2021)

\section{Conclusiones.}

Entre las conclusiones más importantes del presente artículo se puede citar:

- La agricultura se ha convertido en un área esencial de la economía de los países latinoamericanos, cuyos gobiernos deben garantizar la seguridad alimentaria y el cuidado del medio ambiente.

- La propuesta de monitoreo de las políticas agropecuarias en territorios -en concordancia con los objetivos nacionales- tiene como propósito fundamental analizar si estas están enfocadas en revertir las tendencias estructurales del modelo agrario excluyente por un nuevo esquema centrado en tres objetivos estratégicos:

- Contribuir a reducir la pobreza y vulnerabilidad socioeconómica de los habitantes rurales, particularmente, mejorar la inclusión social de aquellos agricultores de pequeña y mediana escala que residen en los territorios.

- Mejorar la contribución de la agricultura para garantizar la seguridad alimentaria y nutricional de la población en cada zona (en el presente y a futuro).

- Potenciar la contribución de la agricultura al desarrollo rural y al crecimiento económico nacional.

- El Ecuador con todos sus entes en el área agropecuaria no están utilizando de manera adecuada el Agrimonitor, herramienta que el BID pone a disposición de los países de la región y que contiene datos de suma importancia para decisiones y políticas agropecuarias que vayan encaminadas en el mejoramiento de las economías de los países de la región.

- El sector agropecuario ecuatoriano ofrece enormes posibilidades para la población y para la economía en su conjunto. Sin embargo, es también un área de gran vulnerabilidad productiva, social y ecológica; por eso la relevancia y preocupación primordial que genera su análisis y atención prioritaria dentro de las políticas públicas. Además de ser un enorme reto, su adecuado tratamiento puede magnificar 
visiblemente la enorme contribución del sector agropecuario, a la inclusión y a la equidad social buscadas.

- Visualizar correctamente sus nudos críticos a través de herramientas como Agrimonitor, identificar las disyuntivas existentes, trazar los objetivos del desarrollo agropecuario y las prioridades, determinar las potencialidades y restricciones constituyen el primer paso de cualquier estrategia para el crecimiento del sector agropecuario y rural en el país.

- Los resultados de las políticas agropecuarias aplicadas en el último medio siglo, obligan a reflexionar sobre transformar la política agropecuaria orientándola hacia un marco de carácter incluyente que respete la soberanía alimentaria, la sostenibilidad ambiental, y considere las necesidades reales de los pequeños y medianos productores campesinos; además de aprovechar el gran potencial agropecuario.

- Los productos agrícolas de exportación del Ecuador equivalen solo el 8\% del PIB, a pesar que productos como el banano, café, cacao, atún, camarón son apreciados en los mercados internacionales, lo cual demuestra que el país tiene un gran potencial de crecimiento en el área agrícola, pero necesita del Estado para que regule políticas agropecuarias que ayuden tanto al consumo nacional como la producción para la exportación.

\section{Referencias Bibliográfica}

Agrimonitor. (2021). www.agrimonitor.iadb.org/es/.

Aguilar, M. (2005). Análisis del desarrollo agrícola en Latinoamérica: Importancia del Capital Social, Estado, Instituciones y Pobreza. Honduras.

ANDREALIZ'S, o. d. (2021). https://andrealiz.wordpress.com/2010/08/27/el-ecuadorprincipales-problemas-en-3-sectores-basicos-agricultura-industria-comerciointernacional/.

Bartra, V. (2002). La protección del medio ambiente y los recursos naturales en la nueva constitución del Perú. Revista Investigación, 9-16.

Carrión, D., \& Herrera, S. (2012). Ecuador Rural del Siglo XXI - Soberania Alimentaria, Inversión Pública y Política Agraria.

CEPAL, C. E. (2018). Perspecivas de la agricultura y del desarrollo rural en las Américas: una mirada hacia América Latina y el caribe 2017-2018.

ELCSA, E. L. (2012). Roma: Organización de las Naciones Unidas para la Alimentación y la Agricultura.

IICA, I. I. (2012). Informe Anual. Ecuador: logros y desafios. Ecuador.

IICA, I. I. (2014). Informe Anual Ecuador: logros.

IICA, I. I. (2019). Informe anual del 2019. Ecuador: logros significativos. Ecuador. 
INEC, I. N. (2021). www.ecuadorencifras.gob.ec/208-seis-cultivos-con-mayorproducción-en-ecuador/. Obtenido de www.ecuadorencifras.gob.ec

MAG, M. d. (2015). El sector agropecuario ecuatoriano: análisis historico y retrospectiva a 2025. En La política agropecuaria ecuatoriana: hacia el desarrollo territorial rural sostenible 2015-2025 I Parte. Quito-Ecuador.

MAG, M. d. (2021). www.agricultura.gob.ec. Obtenido de https://www.agricultura.gob.ec/agricultura-la-base-de-la-economía-y-laalimentación/

Mejia, J. (2004). Sobre la investigación cualitativa: Nuevos conceptos y campos de desarrollo. En Investigaciones sociales (págs. 8-13; 277-299).

PIADAL, P. I. (2013). Agricultura y Desarrollo en América Latina: Gobernanza y políticas públicas. 18-22.

Reinoso, L. (2013). Incidencia de la Agricultura en el Crecimiento y Desarrollo Económico del Ecuador del 2006 al 2021. Quito: Universidad San Francisco de Quito. 


\section{PARA CITAR EL ARTÍCULO INDEXADO.}

Morales Domínguez, A. A., \& Velastegui López, L. E. (2021). Sostenibilidad y Encuentro entre culturas: propuesta semipresencial para guías de turismo. AlfaPublicaciones, 3(3.2), 6-18. https://doi.org/10.33262/ap.v3i3.2.94

\section{Ciencia}

El artículo que se publica es de exclusiva responsabilidad de los autores y no necesariamente reflejan el pensamiento de la Revista Alfa Publicaciones.

El artículo queda en propiedad de la revista y, por tanto, su publicación parcial y/o total en otro medio tiene que ser autorizado por el director de la Revista Alfa Publicaciones.
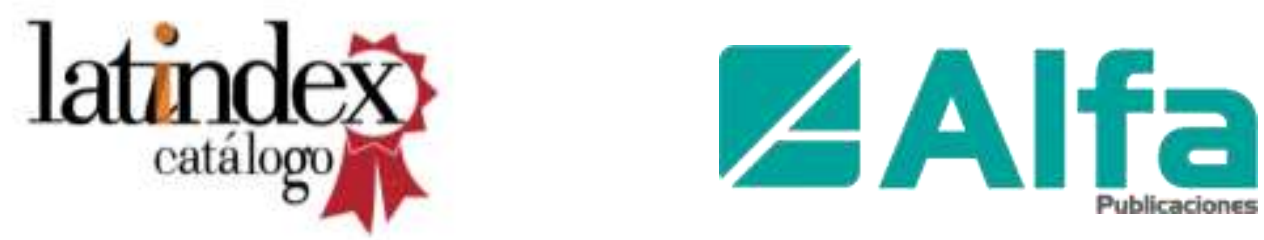